Case

Report

\title{
A Giant Cardiac Cavernous Hemangioma Involving the Left Atrial Roof in an Elderly Woman
}

\author{
Eisaku Nakamura, MD, PhD, ${ }^{1}$ Kunihide Nakamura, MD, PhD,${ }^{1}$ Koji Furukawa, MD, ${ }^{1}$ \\ Hirohito Ishii, MD, ${ }^{1}$ Katsuya Kawagoe, MD, ${ }^{1}$ Takeshi Ideguchi, MD, PhD, ${ }^{2}$ \\ and Nobuyuki Oguri, $\mathrm{MD}, \mathrm{PhD}^{3}$
}

\begin{abstract}
Cardiac tumors are relatively rare, with primary hemangiomas being a particularly rare benign neoplasm. Herein, we report a case of a symptomatic cardiac tumor detected via echocardiography in an 82-year-old woman. Although we performed advanced imaging examinations for her heart, we could not diagnose the tumor before surgery. Eventually, a tumor involving the left atrial roof was detected, and it was completely resected to relieve her symptoms and establish a precise diagnosis. Histopathological examination indicated a cardiac cavernous hemangioma. The patient exhibited an uneventful recovery without any complications.
\end{abstract}

Keywords: atrial roof, cardiac tumor, cardiac cavernous hemangioma, elderly

\section{Introduction}

Cardiac tumors are rare; in a relatively recently reported autopsy series, cardiac tumors were found in only $0.001 \%-0.300 \%$ of cases. ${ }^{1)}$ Among cardiac tumors, primary hemangioma accounted for approximately $2 \%$ of all primary resected tumors, indicating that this is a particularly rare benign neoplasm. Although primary hemangiomas can occur at any age, they occur most commonly in children and adolescents. Primary hemangiomas may

${ }^{1}$ Division of Cardiovascular Surgery, Department of Surgery, University of Miyazaki, Miyazaki, Miyazaki, Japan

${ }^{2}$ Department of Pathology, Faculty of Medicine, University of Miyazaki, Miyazaki, Miyazaki, Japan

${ }^{3}$ Department of Cardiovascular Medicine, University of Miyazaki, Miyazaki, Miyazaki, Japan

Received: July 25, 2017; Accepted: December 7, 2017

Corresponding author: Eisaku Nakamura, MD, PhD. Division of Cardiovascular Surgery, Department of Surgery, University of Miyazaki, 5200 Kihara, Kiyotake, Miyazaki, Miyazaki 889-1692, Japan

Email: ei-naka-1965@mtb.biglobe.ne.jp

(C)2019 The Editorial Committee of Annals of Thoracic and Cardiovascular Surgery. All rights reserved. originate from the endocardium, myocardium, or epicardium and are commonly observed on the free ventricular walls or septum. ${ }^{1,2)}$ Herein, we report a very rare case of a giant cardiac cavernous hemangioma originating from the epicardium of the left atrial roof in an elderly woman.

\section{Case Report}

An 82-year-old woman with a left atrial mass was referred to our hospital by her family doctor. On admission, she complained of dyspnea and bilateral lower extremity edema that had persisted for the past 1 month. Chest radiography revealed cardiac dilatation (cardiothoracic ratio, 58\%), and electrocardiography revealed atrial fibrillation. Transthoracic echocardiography revealed a large mass with an approximate diameter of 8.2 (major axis) $\times 8.0 \mathrm{~cm}$ (minor axis) in the pericardium (Fig. 1). The mass was compressing the left atrial wall. In addition, moderate mitral regurgitation (regurgitant volume, $48 \mathrm{~mL}$; effective regurgitant orifice, $0.31 \mathrm{~cm}^{2}$ ) and pulmonary hypertension with severe tricuspid regurgitation (tricuspid regurgitation pressure gradient, $35 \mathrm{mmHg}$ ) were detected. Bilateral atrial dilatation with a normal left ventricular size and function was observed. 


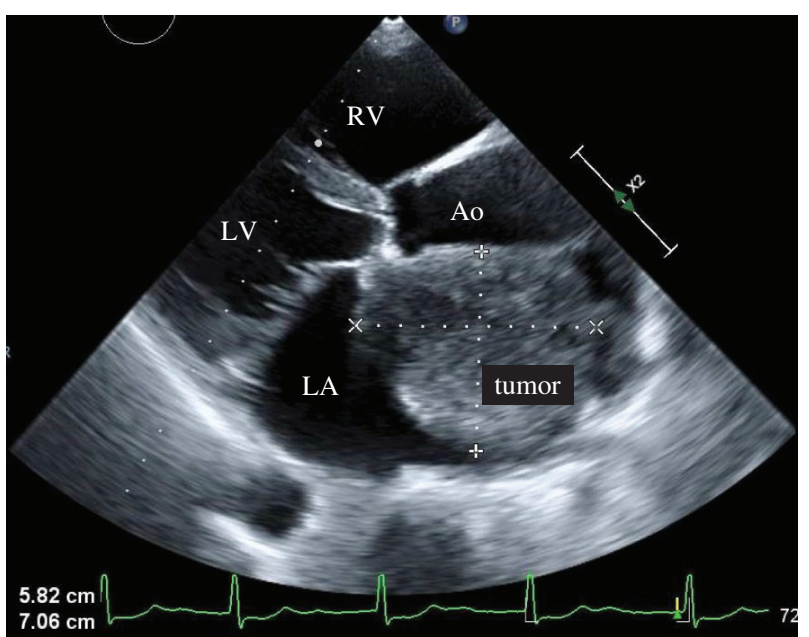

Fig. 1 Transthoracic echocardiography image revealing a large mass close to the roof of the left atrium in the pericardium. The mass measuring 8.2 (major axis) $\times 8.0 \mathrm{~cm}$ (minor axis) in diameter is compressing the left atrial wall. RV: right ventricular; LV: left ventricular; LA: left atrium; Ao: aorta

Contrast-enhanced computed tomography (CT) showed a low-density mass measuring $10.2 \times 6.8 \times 7.1 \mathrm{~cm}$ close to the roof of the left atrium (Figs. 2A and 2B). There was no pericardial effusion. The mass was compressing the left atrial wall and pulmonary artery. Magnetic resonance imaging (MRI) showed a nonhomogeneous signal intensity on T1-weighted images and a high-signal intensity on T2-weighted images (Figs. 2C and 2D). Positron emission tomography (PET)-CT was subsequently performed, which did not reveal fluorodeoxyglucose uptake in the mass. We did not establish a preoperative diagnosis. Surgical resection was scheduled to ameliorate the patient's symptoms and establish a precise diagnosis.

A median sternotomy was employed to access the heart, and the pericardium was opened. The tumor was located on the left atrium; its surface was smooth and no adhesion with the surrounding tissue was observed (Fig. 3A). Cardiopulmonary bypass was achieved via aortic and bicaval cannulation. The aorta was clamped, and cardiac arrest was induced via cold blood cardioplegia. A longitudinal right atriotomy was performed parallel to the sulcus terminalis. Tumor invasion in the right atrium was not observed. Then, we accessed the left atrium via a superior septal approach. Tumor invasion in the left atrium was not observed, and we considered the possibility of a tumor originating from the epicardium. The tumor was gradually peeled off from the external surface of the left atrium wall and completely separated from the left atrium. Therefore, reconstruction of the left atrium wall was not required. Then, mitral valve replacement (CEP magna 27: Edwards Lifesciences; Irvine, CA, USA) was performed because of the extensive degenerative lesion at the anterior leaflet of the mitral valve; moreover, the tumor removal required a lot of time. Tricuspid valve annuloplasty (DeVega operation) was also performed owing to severe tricuspid regurgitation. The patient exhibited an uneventful recovery and was discharged without complications.

The tumor was located at the epicardium, which was partly covered by a single layer of mesothelial cells that demonstrated positivity for calretinin. Histopathological analysis revealed a cavernous vascular tumor with organizing thrombi (Fig. 3B). A final diagnosis of cardiac cavernous hemangioma was established.

\section{Discussion}

We reported a case of a giant cardiac cavernous hemangioma in an elderly woman. Histologically, cardiac hemangioma is generally classified as cavernous (multiple dilated, thin-walled vessels), capillary (small vessels resembling capillaries), or arteriovenous (dysplastic malformation of arteries and veins). ${ }^{1,2)}$ Cardiac hemangioma can occur at any age and may originate from any part of the heart. According to a previous review of 56 cases, cardiac hemangioma occurs most commonly in children and adolescents. In those cases, 20 tumors were located in the right ventricle (37.5\%), 19 in the left ventricle (33.9\%), 13 in the right atrium (23.2\%), 6 in the interatrial septum (10.7\%), 6 in the interventricular septum (10.7\%), and 4 in the left atrium $(7.1 \%){ }^{2)}$ Multiple extensive tumors were noted in 17 cases $(30.4 \%){ }^{2,3)}$ The patient reported here was a very elderly woman, aged 82 years, and her hemangioma originated from the left atrial roof. Considering the site of the tumor and her age, this was considered a rare case.

The symptoms of hemangioma depend on its location and size. While asymptomatic hemangioma has occasionally been reported, ${ }^{4)}$ the lesion can cause dysrhythmia, effusion, heart failure, outflow tract obstruction, and coronary obstruction. ${ }^{2,3)}$ Although hemangiomas are benign, there are reports of sudden death due to benign cardiac tumors ${ }^{5)}$ and life-threatening cardiac tamponade, $\left.{ }^{6}\right)$ which is not always clinically benign. In our case, we believed that the symptoms were caused by bilateral atrial and pulmonary artery compression by the giant hemangioma located close to the left atrial roof.

Cardiac tumors are usually diagnosed via echocardiography, CT, MRI, and/or coronary angiography. 
(A)

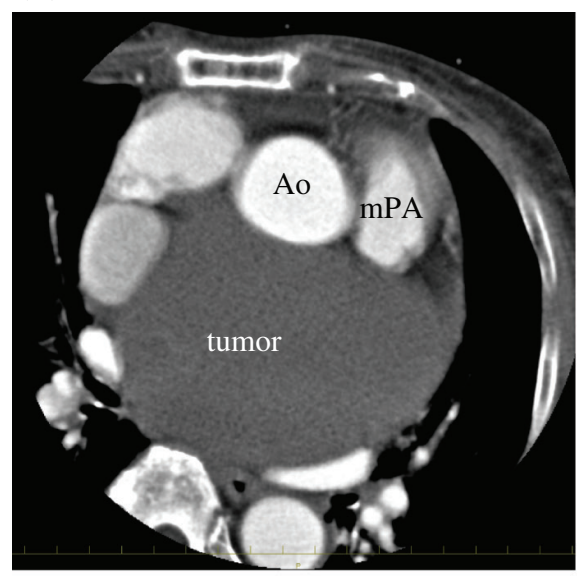

(C)

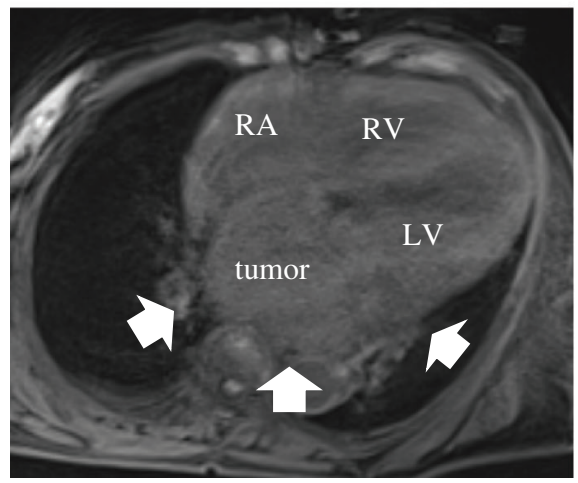

(B)

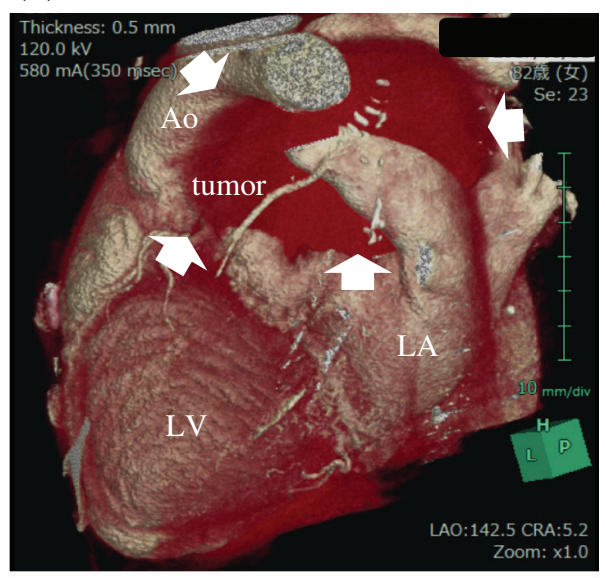

(D)

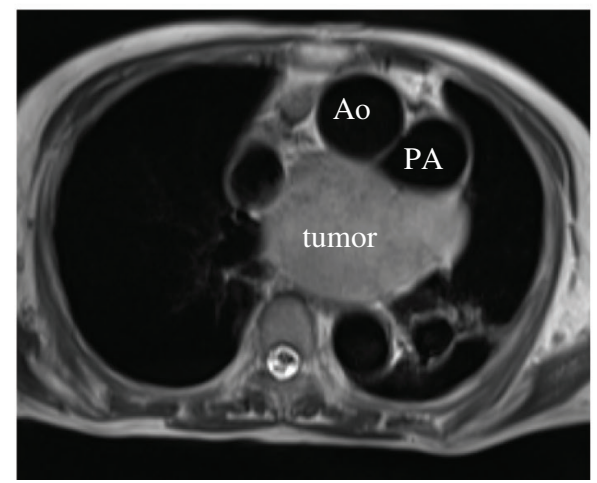

Fig. 2 Contrast-enhanced computed tomography image showing a low-density mass measuring $10.2 \times 6.8 \times 7.1 \mathrm{~cm}$ close to the roof of the left atrium. ([A] coronal view, $[\mathbf{B}]$ threedimensional CT). MRI showing a nonhomogeneous signal intensity on the T1-weighted image (C) and high-signal intensity on the T2-weighted image (D). MRI: magnetic resonance imaging; PA: pulmonary artery; RA: right atrium; RV: right ventricular; LV: left ventricular; LA: left atrium; Ao: aorta; mPA: main pulmonary artery; CT: computed tomography

(A)

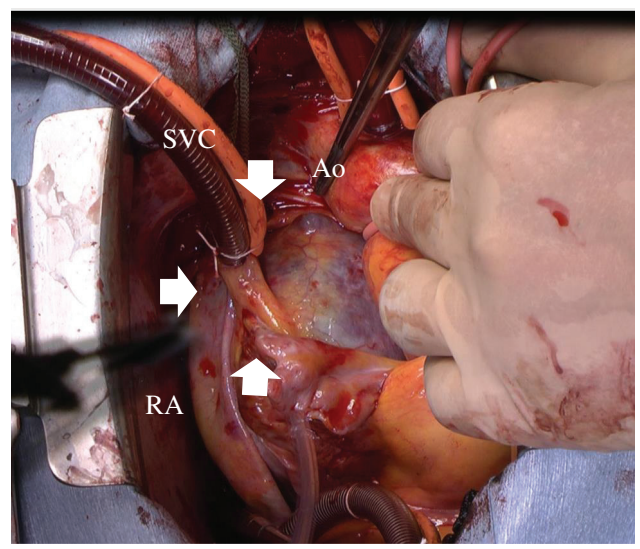

(B)

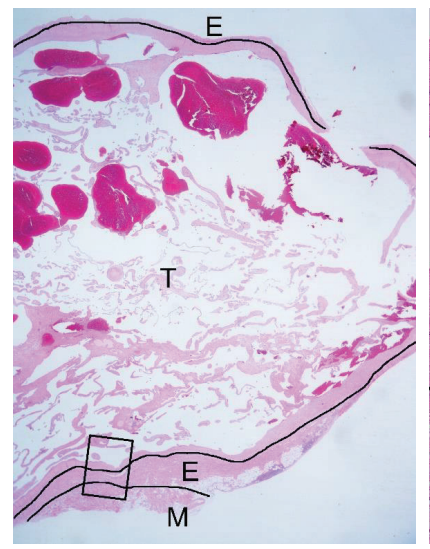

(C)

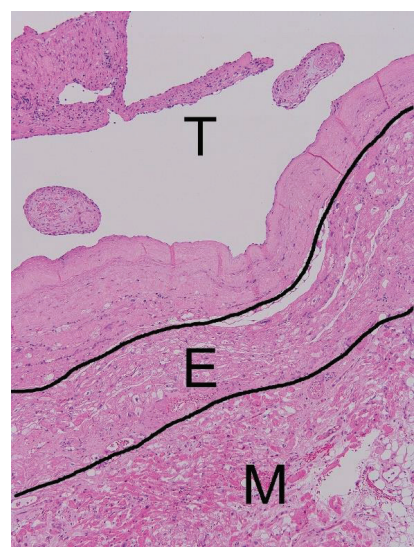

Fig. 3 Intraoperative and histopathological findings. (A): The tumor (arrow) is located on the left atrium, and its surface is smooth. Microscopically, the tumor is composed of cavernous vessels. (B and $\mathbf{C}$ ): The epicardium shows fibrosis and mild chronic infiltration (M: myocardium, E: epicardium, T: tumor) ([B] hematoxylin and eosin staining, $\times 12.5$, $[\mathbf{C}]$ hematoxylin and eosin staining, $\times 100)$. SVC: superior vena cava; Ao: aorta; RA: right atrium 
A preoperative diagnosis is difficult because of the rarity of this lesion. Echocardiography has been proven as the most appropriate screening and diagnostic imaging modality for cardiac tumors. ${ }^{6}$ Contrast-enhanced CT and MRI may identify the vascular origin of a tumor, resulting in the consideration of cardiac hemangioma as a differential diagnosis. Cardiac hemangioma appears as a heterogeneous lesion on non-enhanced chest $\mathrm{CT}$ and, in most cases, an intensely enhanced lesion on contrast-enhanced CT. It typically demonstrates intermediate signal intensity on T1-weighted images and high-signal intensity on T2weighted images. ${ }^{4,7}$ One study reported that PET-CT and angiography are useful for the diagnosis and treatment of cardiac tumors. ${ }^{8)}$ In that study, cardiac hemangiomas exhibited an abnormally increased fluorodeoxyglucose uptake on PET-CT. However, the present case did not show any increase in uptake. Although echocardiography, contrast-enhanced CT, MRI, and PET-CT were performed for the present case, a precise preoperative diagnosis could not be established. A definitive diagnosis of cavernous hemangioma was made only on the basis of a histopathological analysis conducted after surgery.

With regard to cardiac tumors, increasing size, the presence of symptoms, and unclear diagnosis are all indications for surgery. In the present case, cardiac surgery was performed because of the presence of symptoms and an unclear diagnosis. For all cases of hemangioma, complete surgical resection is a standard recommended therapy because unresectable hemangiomas generally confer a poor prognosis. ${ }^{1,49)}$ Whether a tumor is resectable depends on its location and origin. Previous reports have described cases of reconstruction of a left ventricle with a cardiac hemangioma at the apex, ${ }^{10)}$ reconstruction of the right atrium using a bovine pericardial patch, ${ }^{11)}$ and unresectable tumors ${ }^{12)}$ In the present case, it was possible to resect the tumor without reconstructing the left atrium wall. Hemangioma is a disease that can affect all layers of the cardiac wall; in the present case, we believed that resection was possible without cardiac damage because of the involvement of the epicardium. The prognosis was favorable after complete tumor resection. Postoperative follow-up is necessary to monitor recurrence.

\section{Conclusion}

The natural history of primary cavernous hemangioma is unpredictable and largely uncharacterized. The dissemination of information regarding individual cases of hemangioma, such as the one described here, will add to the collective knowledge base pertaining to this tumor.

\section{Acknowledgment}

We would like to thank Editage (www.editage.jp) for English language editing.

\section{Disclosure Statement}

All authors declare that they have no conflicts of interest.

\section{References}

1) Jain D, Maleszewski JJ, Halushka MK. Benign cardiac tumors and tumorlike conditions. Ann Diagn Pathol 2010; 14: 215-30.

2) Kojima S, Sumiyoshi M, Suwa $S$, et al. Cardiac hemangioma: a report of two cases and review of the literature. Heart Vessels 2003; 18: 153-6.

3) Abad C, de Varona S, Limeres MA, et al. Resection of a left atrial hemangioma. Report of a case and overview of the literature on resected cardiac hemangiomas. Tex Heart Inst J 2008; 35: 69-72.

4) Thomas JE, Eror AT, Kenney M, et al. Asymptomatic right atrial cavernous hemangioma: a case report and review of the literature. Cardiovasc Pathol 2004; 13: 341-4.

5) Cina SJ, Smialek JE, Burke AP, et al. Primary cardiac tumors causing sudden death: a review of the literature. Am J Forensic Med Pathol 1996; 17: 271-81.

6) Lo LJ, Nucho RC, Allen JW, et al. Left atrial cardiac hemangioma associated with shortness of breath and palpitations. Ann Thorac Surg 2002; 73: 979-81.

7) Li ZY, Li XC, Wu Q. PET-CT diagnosis of cardiac cavernous hemangioma with large pericardial effusion. Eur Rev Med Pharmacol Sci 2014; 18: 3256-9.

8) Grebenc ML, Rosado de Christenson ML, Burke AP, et al. Primary cardiac and pericardial neoplasms: radiologic-pathologic correlation. Radiographics 2000; 20: 1073-103; quiz 1110-1, 1112.

9) Unger E, Costic J, Laub G. Giant Cardiac Cavernous Hemangioma. Ann Thorac Surg 2015; 100: 322-5.

10) Tomizawa $Y$, Endo M, Nishida $H$, et al. Reconstruction of the left ventricle in a patient with cardiac hemangioma at the apex. Ann Thorac Surg 2001; 71: 2032-4.

11) Yuan SM, Shinfeld A, Kuperstein R, et al. Cavernous hemangioma of the right atrium. Kardiol Pol 2008; 66: 974-6.

12) Botha J, Ihlberg L, Elhenawy A, et al. A giant cavernous hemangioma of the heart. Ann Thorac Surg 2010; 90: $293-5$. 